\title{
Infection Dynamics in Coexisting Sexual and Asexual Host Populations: Support for the Red Queen Hypothesis*
}

\author{
Daniela Vergara, ${ }^{1}$ Jukka Jokela, ${ }^{2}$ and Curtis M. Lively ${ }^{1, \dagger}$
}

1. Department of Biology, Indiana University, Bloomington, Indiana 47405; 2. EAWAG, Swiss Federal Institute of Aquatic Science and Technology, Überlandstrasse 133, CH-8600 Duebendorf, Switzerland; and ETH Zurich, Swiss Federal Institute of Technology, Institute of Integrative Biology, 8092 Zurich, Switzerland

Dryad data: http://dx.doi.org/10.5061/dryad.29nk3.

Dryad data: http://dx.doi.org/10.5061/dryad.40d6h.

ABSTRACT: The persistence of sexual reproduction is a classic problem in evolutionary biology. The problem stems from the fact that, all else equal, asexual lineages should rapidly replace coexisting sexual individuals due to the cost of producing males in sexual populations. One possible countervailing advantage to sexual reproduction is that, on average, outcrossed offspring are more resistant than common clones to coevolving parasites, as predicted under the Red Queen hypothesis. In this study, we evaluated the prevalence of infection by a sterilizing trematode (Microphallus sp.) in a natural population of freshwater snails that was composed of both sexual and asexual individuals (Potamopyrgus antipodarum). More specifically, we compared the frequency of infection in sexual and asexual individuals over a 5-year period at four sites at a natural glacial lake (Lake Alexandrina, South Island, New Zealand). We found that at most sites and over most years, the sexual population was less infected than the coexisting asexual population. Moreover, the frequency of uninfected sexual females was periodically greater than two times the frequency of uninfected asexual females. These results give clear support for a fluctuating parasite-mediated advantage to sexual reproduction in a natural population.

Keywords: asexual reproduction, host-parasite coevolution, infection dynamics, Potamopyrgus antipodarum, Red Queen hypothesis, sexual reproduction.

\section{Introduction}

The coexistence of sexual and asexual lineages of the same species is difficult to explain. All-female, asexual lineages should (all else equal) exhibit higher per capita birth rates (Maynard Smith 1971, 1978) and higher carrying capacities (Doncaster et al. 2000; Lively 2009) than sexual lineages. Hence, asexuals should rapidly replace co-occurring sexuals.

\footnotetext{
* This issue originated as the 2013 Vice Presidential symposium presented at the annual meetings of the American Society of Naturalists.

$\dagger$ Corresponding author; e-mail: clively@indiana.edu.
}

Am. Nat. 2014. Vol. 184, pp. S22-S30. (C) 2014 by The University of Chicago. 0003-0147/2014/184S1-54851\$15.00. All rights reserved. DOI: $10.1086 / 676886$
Why, then, does sex exist? How do sexual and asexual forms of the same species coexist in space and time?

The Red Queen hypothesis offers a promising explanation for the coexistence of sexual and asexual forms in space and time. Under this hypothesis, parasite-mediated selection against common host genotypes leads to the maintenance of genetic diversity for disease resistance in sexual populations (Haldane 1949). If parasites are common and virulent, then parasite-mediated selection may be strong enough to prevent the fixation of common clones in mixed (sexual and asexual) populations (Jaenike 1978; Lloyd 1980; Bell 1982; Hamilton 1982). This negative frequency-dependent selection against clonal lineages can lead to the stable coexistence of sexual and asexual individuals that compete for the same resources (Howard and Lively 1994; Lively 2009, 2010). Similarly, theory suggests that parasite-mediated selection against common genotypes can indirectly select for intermediate rates of recombination (Peters and Lively 1999, 2007; Schmid-Hempel and Jokela 2002; Gandon and Otto 2007), and lead to stable mixtures of self-fertilizing and cross-fertilizing individuals (i.e., mixed mating; Agrawal and Lively 2001).

Support for the Red Queen hypothesis comes from a variety of sources. First, biogeographic surveys have shown that asexual reproduction dominates in populations where parasites are relatively rare (Lively 1992; Verhoeven and Biere 2013), even at small spatial scales (Vergara et al. 2013). These results suggest that asexuals eliminate sexuals where parasite-mediated selection is weak. Second, parasites can exhibit a high degree of genetic specificity for infection (Rauch et al. 2006; Dybdahl et al. 2008; Wegner et al. 2008; Wilfert and Schmid-Hempel 2008; Duneau et al. 2011; Luijckx et al. 2011, 2013), which is a necessary condition for parasite-mediated selection against common genotype. Third, parasite-mediated selection against common clones has been observed in laboratory experiments (Koskella and Lively 2009) as well as in the field for a wide 
range of taxa (Antonovics and Ellstrand 1984; Kelley et al. 1988; Lively et al. 1990; Chaboudez and Burdon 1995; Dybdahl and Lively 1998; Lively and Dybdahl 2000; Michiels et al. 2001; Jokela et al. 2009; Wolinska and Spaak 2009; Šimková et al. 2013). Fourth, time-shift experiments (wherein hosts are exposed to parasites that were "frozen" from different time periods) have shown that parasites rapidly track common host genotypes in a variety of microbes, plants, and animals (Buckling and Rainey 2002; Decaestecker et al. 2007; Koskella and Lively 2009; Thrall et al. 2012; Koskella 2013, 2014). Fifth, ancient asexual reproduction appears to be evolutionarily stable in rotifers, owing at least in part to the fact that the rotifers disperse farther than their parasites, thereby preventing antagonistic coevolution (Wilson and Sherman 2010, 2013). Sixth, recent laboratory experiments have shown the evolution of increased rates of outcrossing in hermaphroditic worms when coevolving with virulent pathogens (Morran et al. 2011, 2013) and that outcrossed offspring were more resistant to infection than self-fertilized offspring (Masri et al. 2013). Finally, experimental work has shown that antagonistic coevolution can lead to increased rates of recombination in flour beetles (Fischer and Schmid-Hempel 2005; Kerstes et al. 2012).

In this work, we build on these previous studies by testing the hypothesis that parasite-mediated selection against asexual reproduction can generate an overall advantage to sexual reproduction in a natural population. Specifically, using long-term field data, we compared the prevalence of sterilizing infections in coexisting sexual and asexual females in a natural lake population of freshwater snails (Potamopyrgus antipodarum). Under the Red Queen hypothesis for the maintenance of sex, we predicted that sexual females would, on average, be less infected than asexual females.

Previous work in this system has shown a significant, positive association between parasites and the occurrence of sexual reproduction in the snail. These studies were conducted across multiple lake populations (Lively 1992; Lively and Jokela 2002). Across populations, the Red Queen hypothesis predicts that asexuals would outcompete sexuals where the strength of parasite-mediated selection is weak, thus generating a positive overall relationship between sex and infection among populations. However, within populations, where sexual and asexual females compete directly for resources, the Red Queen hypothesis predicts that asexual females should be more infected over time than sexual females.

Previous studies of this snail-trematode interaction have also demonstrated rapid evolution by the parasite (a trematode worm, Microphallus sp.) to infect common asexual clones both in the field (Dybdahl and Lively 1998; Jokela et al. 2009) and in controlled laboratory experiments (Koskella and Lively 2009). Overinfection of common clones in these studies was correlated with a turnover in clonal composition, such that rare clones increased in frequency over time. Moreover, laboratory studies have shown that sexual populations evolve resistance in response to infection and that resistance is specific to the parasite source (Koskella et al. 2011).

These prior results meet necessary, but not sufficient, conditions for a parasite-mediated advantage to sexual reproduction in P. antipodarum. Hence, in this study, we compared the annual prevalence of infection in sexual and asexual snails to determine whether host-parasite coevolution could generate an overall advantage to sexual reproduction. By averaging across the fecundity differences for a diverse set of clones (Jokela et al. 2003; Paczesniak 2012), we were able to calculate the overall parasite-mediated advantage to sexual reproduction over asexual reproduction. The data were collected over a 5-year period at four sites in a natural lake population of snails. Consistent with the Red Queen hypothesis, the results showed strong parasite-mediated selection against asexual reproduction.

\section{Methods}

\section{Natural History of the Study System}

Potamopyrgus antipodarum is a small (4-5 mm), ovoviviparous freshwater snail that is common in subalpine lakes and streams throughout New Zealand. Many populations of this snail contain diploid sexual females (and diploid males) living in coexistence with triploid asexual females (Phillips and Lambert 1989; Wallace 1992; Dybdahl and Lively 1995). Where they coexist, sexual and asexual females mature at the same size and carry the same number of embryos in their brood pouches on average. Along with laboratory experiments, these findings demonstrate that there is a cost to producing males in the sexual population (Jokela et al. 1997). Finally, the broods of sexual females tend to have multiple sires as well as a high evenness of success among sires, which would tend to increase the genetic diversity of sexual offspring (Soper et al. 2012).

Lake populations of the snail range across three major depth-stratified habitat zones: shallow $(<1 \mathrm{~m})$; mid depth, which tends to be dominated by plants in the genus Isoetes (1-3 m); and a deep zone $(4-7 \mathrm{~m})$, which tends to be dominated by larger macrophytes such as native Myriophyllum sp., Chara sp., Nitella sp., and invasive Elodea canadensis. Snail density is usually very high (e.g., $>1,000$ $\mathrm{m}^{-2}$ in the mid-depth zone; Paczesniak et al. 2014). In addition, we have found that different clones tend to dominate the different habitats, suggesting that snails do not move freely among zones (Fox et al. 1996; Jokela et al. 1999). At our primary study site, Lake Alexandrina (South 
Island, New Zealand), sexual and asexual snails coexist, but there is a striking cline from the shallow to deep habitats. Sexual snails are most common in the shallow zone, while asexuals dominate in the deep zone. The mid-depth zone contains an approximately equal mixture of sexual and asexual snails (Jokela and Lively 1995a, 1995b).

The snail serves as the first-intermediate host for at least 20 species of trematodes for which the sterilizing trematode Microphallus sp. "livelyi" (Hechinger 2012) is the most common in lake populations of the snail (Winterbourn 1974; Lively 1987). Like most trematodes, Microphallus has a complex life cycle but with some rather unique features. The adult worms are small $(<0.5 \mathrm{~mm})$, outcrossing, simultaneous hermaphrodites that live in the intestines of ducks. Eggs produced by the parasite are shed with the duck's feces. The eggs hatch following ingestion by the snail host, and successful infections result in the asexual production of thousands of larvae (metacercariae) that encyst in the snail (K. Klappert and J. Jokela, unpublished data). The infection completely sterilizes both males and females, and it cannot be cleared by the snail. Hence, the parasites are highly virulent in terms of their effect on snail fitness.

The larval cysts of this trophically transmitted parasite hatch in the stomachs of waterfowl. Thus, waterfowl must consume live, infected snails for the parasite's life cycle to be completed. As such, the distribution of this parasite is determined by the distribution and foraging habits of New Zealand waterfowl. At our study sites, grey ducks, introduced mallards, and their hybrids tend to forage in the shallow-water margins of lakes $(<1 \mathrm{~m}$ deep), while New Zealand scaup will also forage in the mid-depth region (2-3 m deep; J. Jokela and C. M. Lively, personal observations). Hence, infections in the deep zone $(>4 \mathrm{~m})$ are unlikely to be transmitted to ducks. Consistent with this expectation, experiments have shown that the parasite is adapted to infect sympatric snails from the shallow-water habitat but not from the deepwater habitat (King et al. 2009, 2011). In fact, Microphallus were no more infective to sympatric, deepwater snails than they were to allopatric snails, suggesting that the deepwater habit is a coevolutionary cold spot (King et al. 2009, 2011).

\section{Field Collections}

The study was conducted using snails collected over a 5year period at Lake Alexandrina, where sexual and asexual snails are known to coexist in the shallow-water margins. Lake Alexandrina is a glacial lake in the McKenzie Basin, an intermountain basin in the South Island of New Zealand; it is also a waterfowl preserve. The lake is located at an altitude of $730 \mathrm{~m}$ with a maximum width of $0.9 \mathrm{~km}$ and a maximum length of $7.2 \mathrm{~km}$. We collected large $(N>200)$, random samples of snails during January and February of 2001 to 2005 from the shallow-water habitat at four sites on the southwest side of Lake Alexandrina (Camp, Swamp, West Point, and a fourth site called Halfway, in between Second Fence and West Point (see Jokela and Lively $1995 b$ for a detailed map and collection methods; see also http://www.ae.ethz.ch/research/potamo/alex_map). The snails were transported to the Edward Percival Field Station in Kaikoura, New Zealand where they were sieved with a 2-mm sieve to exclude juveniles. Each individual snail was then measured, sexed, and dissected. The prevalence of Microphallus infection was determined with the aid of a dissecting microscope. Head tissue from each snail was frozen and preserved in an $-80^{\circ} \mathrm{C}$ freezer.

\section{Determination of Reproductive Mode}

In order to determine whether asexual snails were more or less infected than sexual snails, we performed flow cytometry on frozen head tissue at the Indiana University Flow Cytometry Facility. Because asexual snails are triploid and sexual snails are diploid, we were able to determine the reproductive mode of each individual snail by quantifying the amount of its DNA by flow cytometry (Neiman et al. 2011). Flow cytometry was conducted using the BD Biosciences FACSCalibur machine, which has a two-laser analyzer, and CellQuest Pro software. Each frozen sample was ground, filtered, and treated with propidium iodide, which binds to the DNA and stains the cells (Osnas and Lively 2006). We used the FL2 channel to assess propidium iodide fluorescence and DNA content. At the beginning of each run, we calibrated the machine using known sexual (diploid) and asexual (triploid) snails in order to define "gates," which were used to designate snails as either asexual triploids or sexual diploids (Neiman et al. 2011). For each run, we randomly chose one sexual standard from a lab-reared sexual population originally from Lake Alexandrina. For the asexual standards, we used two snails from a clonal, lab-reared lineage that also originated from Lake Alexandrina. At least 5,000 cells were measured for each sample (Osnas and Lively 2006). The asexual genome has approximately $1,350 \mathrm{mbp}$, while the sexual genome has approximately $900 \mathrm{mbp}$ (Neiman et al. 2011). Therefore DNA quantity in the FL2 channel for propidium iodide ranged from 482.83 to 794.48 with a mean of 659.25 for the asexuals and from 283.36 to 464.29 with a mean of 387.52 for the sexuals. The individuals that fell in between the sexual or asexual gate were categorized as unknown and excluded from the analysis $(N=27$ individuals from all years and all sites). Because we only used the heads of snails for flow cytometry, we excluded the possibility of contamination from parasite DNA, which would generate a separate peak (Neiman et al. 2011). 
For each run, we analyzed 36 individuals, including the three standard snails. For every run, we examined the snails collected in a single year from a single site, and then we continued with different sites and years until we had analyzed all sites and all years. We repeated this process until we had sample sizes of 316, 307, 338, and 218 females for Camp, Halfway, Swamp and West Point, respectively.

\section{Statistical Analyses}

In order to test for differences in the prevalence of $\mathrm{Mi}$ crophallus infection between diploid and triploid females as well as for differences among sites and among years, we analyzed the data using a generalized linear model (GLM) with binomial errors and logit link function. Males were excluded from the original analyses, as infection in males would not affect the per capita birth rate of the sexual population, unless sexual females became sperm limited. Nonetheless, including males in later analyses did not qualitatively change the results. We did not fit the three-way interaction term between site, year, and ploidy, because, at one site (West Point), asexual females were absent in the samples for the last 2 years of the study. We also used a GLM to test whether the frequency of sexual females varied according to year and site. As in the previous analysis, it was not possible to fit the interaction between year and site in the analysis for frequency of sexual females because of the absence of asexual females at West Point in 2004 and 2005. With both GLMs, we used a repeated contrast to examine whether changes in prevalence of infection or frequency of sexual females between consecutive years were statistically significant. Repeated contrasts compared the mean of each year to the mean of next year and are generally used in profile analysis or where difference scores are examined (Norusis 1994).

Finally, to further understand the coexistence of sexual and asexual snails, we estimated the geometric mean fitness of both the asexual and the sexual populations over time. First, we estimated the fitness of the sexual population $\left(W_{\text {sex }}\right)$ for each year as $p^{\prime} / p$, which is the frequency of sexuals in year $i+1\left(=p^{\prime}\right)$ divided by the frequency of sexuals in year $i(=p)$. Similarly, we estimated the fitness of the asexual population $\left(W_{\text {asex }}\right)$ for each year as $q^{\prime} / q$. Following Orr (2009), we calculated the geometric mean fitness over time for the sexual population as $G_{\text {sex }}=$ $\bar{W}_{\text {sex }}-\left(\sigma_{\text {sex }}^{2} / 2 \bar{W}_{\text {sex }}\right)$, where $\left(\bar{W}_{\text {sex }}\right)$ is the arithmetic mean of the fitness estimates for the sexual population over all years and $\sigma_{\text {sex }}^{2}$ is the variance in fitness over time. Similarly, the geometric mean fitness for the asexual population was estimated as $G_{\text {asex }}=\bar{W}_{\text {asex }}-\left(\sigma_{\text {asex }}^{2} / 2 \bar{W}_{\text {asex }}\right)$. If, over time, a parasite-mediated advantage to outcrossing can balance the cost of males, the geometric means for the sexual and asexual subpopulations should be approximately equal.

\section{Results}

We found that the prevalence of infection in both sexual and asexual females changed dramatically over the 5-year period of the study (table 1; fig. 1A). The overall mean prevalence of infection declined significantly between 2001 and 2002 (repeated contrast, Wald $\chi^{2}=9.42, P=.002$ ), and then increased significantly between 2003 and 2004 (Wald $\chi^{2}=4.74, P=.029$ ). The changes in prevalence between 2002 and 2003 (Wald $\chi^{2}=0.30, P=.589$ ) and between 2004 and 2005 (Wald $\chi^{2}=0.61, P=.435$ ) were not statistically significant. We also observed highly significant year, site, and year-by-site interactions (table 1; fig. 2), which are suggestive of complex spatiotemporal variation in coevolutionary interactions (Thrall et al. 2012).

Most importantly, sexual females were, on average, significantly less infected than asexual females (see "Reproductive mode" in table 1). The magnitude and direction of the difference in infection depended significantly on year (fig. $1 A$; also see: "Y $\times \mathrm{R}$ " interaction in table 1 ). Repeated contrasts analysis by reproductive mode showed that asexual females were more infected than sexual females from 2001 to 2004 and that the difference was statistically significant in 2001, 2002, and 2004 (fig. 1A). During the last year of the study (2005), the pattern reversed: sexual females had a significantly higher prevalence of infection than asexual females (fig. 1A). This latter result indicates that diploids are not inherently more resistant to infection than triploids and that the parasite-mediated advantage of sexual reproduction can fluctuate through time.

To determine the impact of infection on the relative fitness of sexuals and asexuals, we divided the proportion of uninfected sexual females by the proportion of uninfected asexual females (fig. 3). We found that the proportion of uninfected sexual females was periodically more than double the proportion of uninfected asexual females.

Table 1: ANOVA in the prevalence of Microphallus infection by year (2001-2005), site (four shallow sites of Lake Alexandrina), and reproductive mode (asexual females vs. sexual females)

\begin{tabular}{lrrr}
\hline Effect & Wald $\chi^{2}$ & df & $P$ \\
\hline Intercept & 1.226 & 1 & .268 \\
Year (Y) & 15.943 & 4 & .003 \\
Site $(S)$ & 22.873 & 3 & $<.001$ \\
Reproductive mode $(\mathrm{R})$ & 6.782 & 1 & .009 \\
Y $\times$ S & 90.183 & 12 & $<.001$ \\
Y $\times \mathrm{R}$ & 22.883 & 4 & $<.001$ \\
$\mathrm{~S} \times \mathrm{R}$ & 5.639 & 3 & .131 \\
\hline
\end{tabular}

Note: The model was fitted using a generalized linear model with a binomial error distribution and logit link function. The three-way interaction of year $\times$ site $\times$ reproductive mode was not fitted due to the absence of asexual females at one site (West Point) for the last 2 years of the study. The data underlying this table and figures 1-3 are deposited in Dryad Digital Repository: http://dx.doi.org/doi:10.5061/dryad.29nk3 (Vergara et al. 2014). 

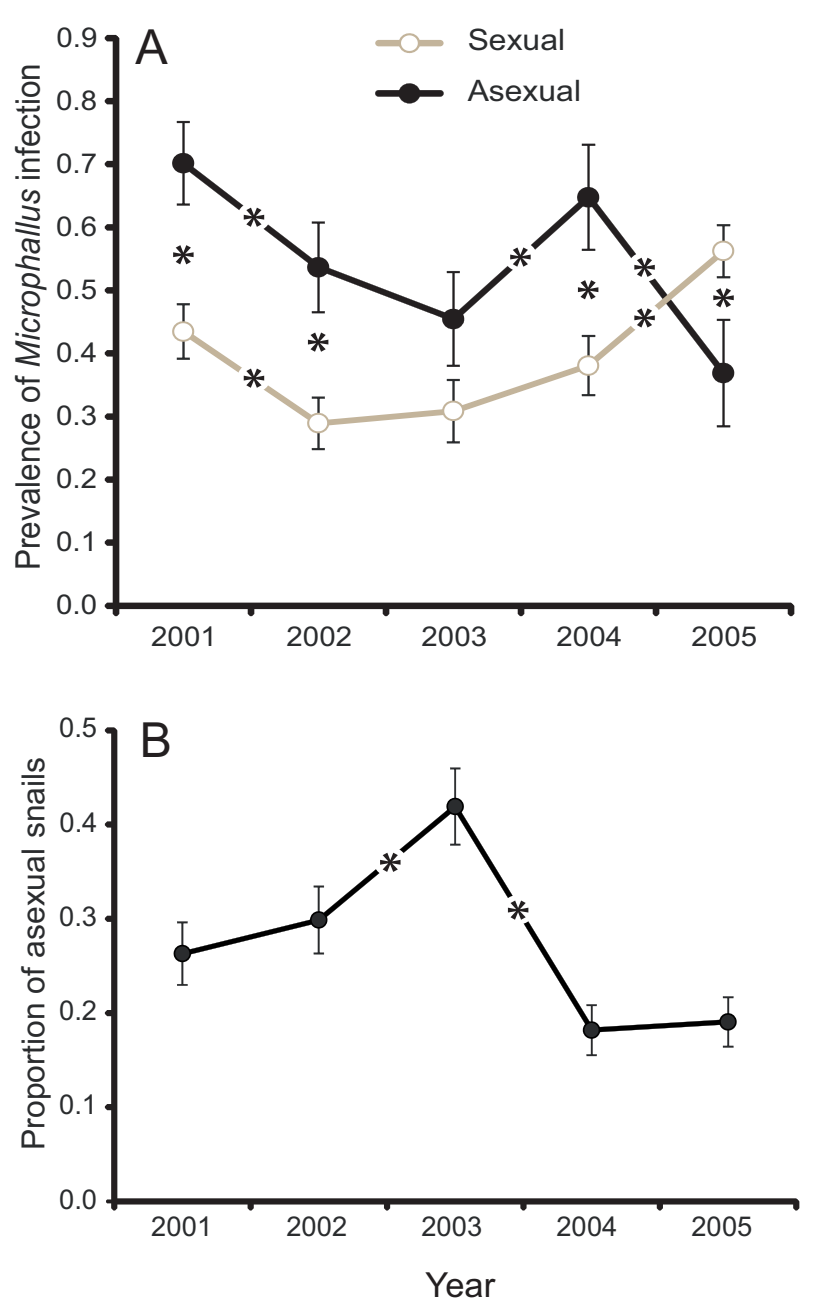

Figure 1: Prevalence of infection and the proportion of asexual females over time. A, Mean prevalence of Microphallus infection ( \pm SE) for sexual and asexual female snails averaged over all sites during the study period. Asterisks denote statistical significance of specific hypotheses. Asterisk overlying the line show results of repeated contrasts for each reproductive mode (asexual and sexual, respectively). For sexual and asexual females, we observed a change in the prevalence between 2001-2002 and 2004-2005 that was statistically significant at the 5\% level. Similarly, change in prevalence between 2003 and 2004 was statistically significant for asexuals. Asterisks between means of sexual and asexual samples indicate those years where the difference in prevalence between sexual and asexual females was significant at the $5 \%$ level when tested with a pairwise contrast (years $2001,2002,2004$, and 2005). B, The proportion of female snails that were asexual over time, averaged over all four sites.

Given that only uninfected females are reproductive, this result suggests that parasite-mediated selection confers a periodic advantage to sexual reproduction that could overcome even a full, twofold cost of producing males.

The proportion of asexual females increased between 2001 and 2003 from $25 \%$ to over $40 \%$ and then declined rapidly between 2003 and 2004 to level off below 20\% during 2004-2005 (fig. 1B). Interestingly, the peak infection frequency in the asexual population occurred just 1 year after the peak in the proportion of asexual individuals in 2003 (cf. fig. 1A, 1B). Repeated contrasts suggest that the changes in the proportion of asexuals between 20022003 and 2003-2004 were statistically significant. The overall variance among years was highly significant in the analysis (Wald $\chi^{2}=41.66$, $\mathrm{df}=4, P<.001$ ), as was the variation among sampling sites (Wald $\chi^{2}=84.40$, $\mathrm{df}=$ $3, P<.001$; see fig. $1 B$ ). Sparse data at West Point for years 2004 and 2005 did not allow us to fit the interaction term between year and site, but if only three sites (Camp, Swamp and Halfway) were included in the analysis, the interaction between year and site was statistically significant (Wald $\chi^{2}=16.78, \mathrm{df}=8, P=.032$ ).

Males were excluded from the analyses presented above, as infection in males does not affect the per capita birth rate of the sexual population. However, inclusion of males in the analyses did not alter our results. Moreover, we found no significant difference between the mean infection rates of males $(\overline{\mathrm{X}}=0.38)$ and diploid females $(\overline{\mathrm{X}}=0.40)$ over the 5 years of the study (Wald $\chi^{2}=0.398, \mathrm{df}=1$, $P=.528)$.

Taken together, our results show a parasite-mediated advantage to sexual reproduction for 4 of the 5 years of the study. This parasite-mediated advantage is of sufficient magnitude to counterbalance the cost of producing males and thus could explain the observed coexistence of sexual and asexual morphs. Consistent with coexistence, our estimates of the geometric mean fitness were not significantly different between sexual and asexuals: $G_{\text {sex }}=1.01, G_{\text {asex }}=$ 0.94 ( $t$-test, $P=.71$ ), indicating that neither reproductive strategy had an overall advantage during the course of the study.

\section{Discussion}

The primary goal of our study was to determine whether asexual females are, on average, more infected than sexual females, as expected under the Red Queen hypothesis. To this end, we studied a natural population of freshwater snails where long-term data suggests stable coexistence of sexual and asexual individuals (Jokela et al. 2009). We found that, averaged across all years and sites, asexual females were indeed significantly more likely to be infected by a common, sterilizing trematode worm than were sexual females (fig. 1). Moreover, we found that the frequency of uninfected sexual females was more than twice the frequency of uninfected asexual females at two of three sites for some years of the study, and 1.5 times greater at a third site (fig. 3). Because only uninfected females are capable of reproduction, these results strongly suggest that 

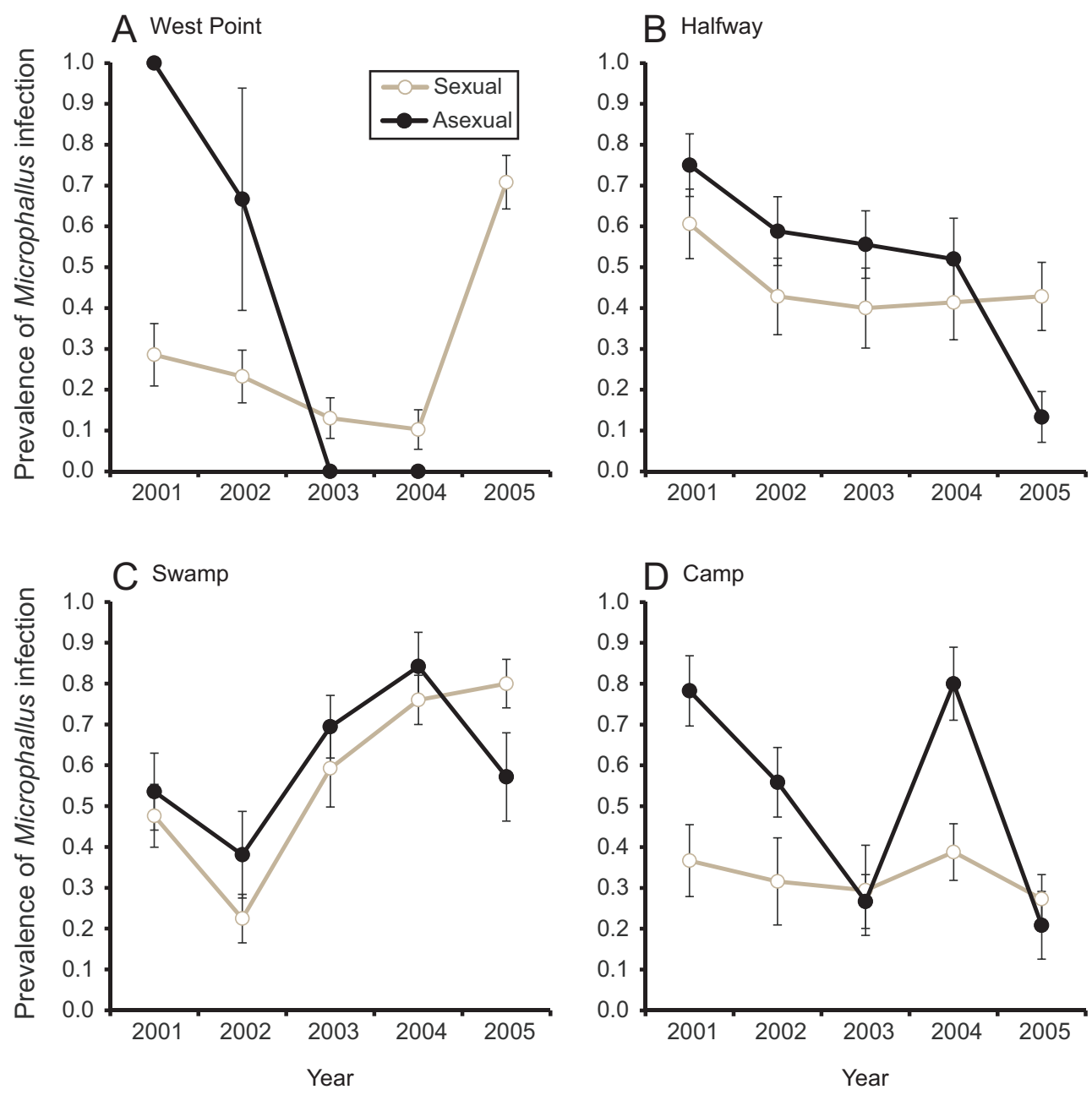

Figure 2: Percent infection in sexual and asexual females for four sites. Percent infection of sexual (open circles) and asexual (filled circles) females for each site West Point $(A)$, Halfway $(B)$, Swamp $(C)$, and Camp $(D)$.

infection by these parasites results in a spatiotemporal advantage to sexual reproduction that is sufficient to overcome the cost of producing males.

In addition, we found that sexual females were significantly more infected than asexual females during the last year of the study. Hence, it would appear that sexual snails are not inherently more resistant to infection and that parasite-mediated selection can periodically favor asexual reproduction. Nonetheless (as indicated above), averaged over all years and sites, asexuals were more likely to be infected than sexuals (figs. 1,2). The fluctuating advantage to sexual reproduction caused by parasites may allow for the coexistence of sexual and asexual individuals. Along these lines, we found that the geometric mean fitness' of the sexual and asexual populations were not significantly different, suggesting that parasites may contribute to the long-term stable coexistence of sexual and asexual forms in this population.

These results are consistent with previous studies at Lake Alexandrina. The host-parasite community at this lake has been particularly useful for addressing questions related to the evolutionary maintenance of sex, because sexual and asexual females coexist in the shallow-water habitats, where infection by trematodes is relatively high, and asexuals dominate in the deeper regions of the lake, where infection is very low. Previous studies on this system have shown that the parasite population is significantly better adapted to infect shallow-water snails than deepwater snails, suggesting that the shallow-water region of the lake is a coevolutionary "hotspot" (in the sense of Thompson 1994, 2005), while the deep part of the lake ( $>4 \mathrm{~m}$ deep), where the asexuals dominate, is a coevolutionary 


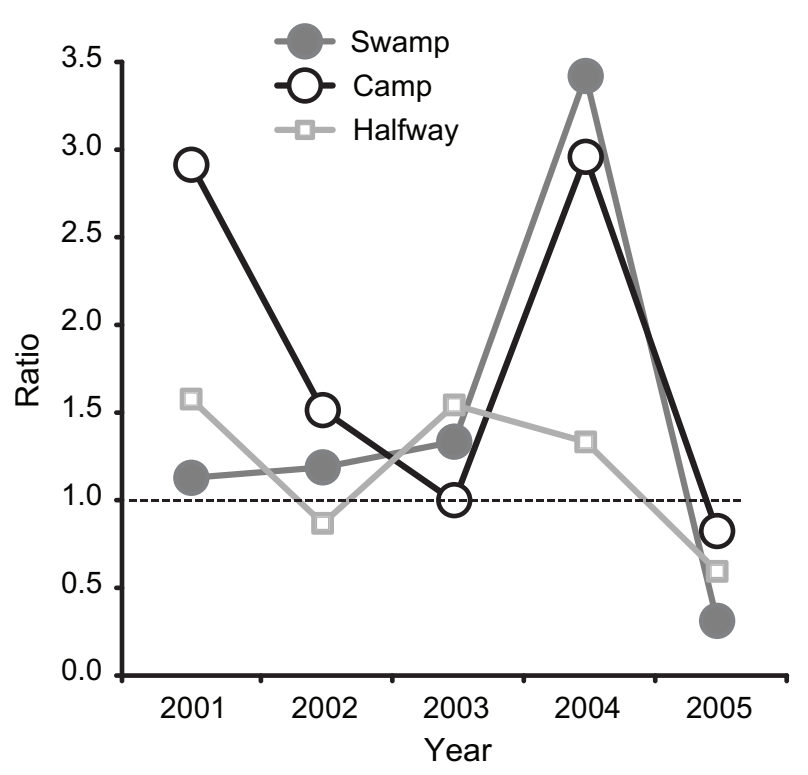

Figure 3: The proportion of uninfected sexual females divided by the proportion of uninfected asexual females. This ratio is shown for three sites (Swamp, filled cirlces; Camp, open circles; and Halfway, open squares). One site (West Point) had to be excluded, as it did not contain asexual females for some years (fig. 3). The dashed line gives a $1: 1$ ratio.

"coldspot" (King et al. 2009, 2011). This finding makes sense, because the final hosts (dabbling ducks and New Zealand scaup) do not forage in the deeper parts of the lake. In addition, a recent study found that the clonal composition of the deep population is relatively stable, at least when compared to the rapid turnover of clonal genotypes in the shallow-water population (Paczesniak et al. 2014). For example, between 2003 and 2007, there was a dramatic reduction in clonal diversity and evenness in the shallow habitat. In fact, by 2007 , the shallow-water population of asexual snails had become dominated by a single clone (Paczesniak et al. 2014), which was highly resistant to infection by Microphallus (Paczesniak 2012). Importantly, the changes in clonal structure observed by Paczesniak et al. (2014) occurred exactly during the period for which we observed a significant change in relative infection rates of sexual and asexual snails (fig. 1, years 20042005). Thus, it is likely that the asexual subpopulation became less infected than the sexual population in 2005 as a consequence of the spread of this resistant clone.

Taken together, our results are consistent with a growing number of empirical studies that support the basic tenets of the Red Queen hypothesis. For example, in Daphnia, common clones decreased in frequency over time in lakes where parasites were observed but not in lakes where parasites were not found (Wolinska and Spaak 2009). Simi- larly, a common Daphnia clone became highly susceptible to infection during the course of an epidemic and then dramatically declined in frequency (Duncan and Little 2007). In addition, time-shift experiments with Daphnia and its parasites collected from sediment cores showed strong evidence of parasites tracking locally common clonal host lineages in a field population (Decaestecker et al. 2007). Conceptually similar time-shift experiments using a plant-fungus (Linum-Melampsora) interaction revealed strong evidence of parasite-mediated selection against common resistance genotypes in the host plants as well as complex spatiotemporal patterns of selection in the metapopulation (Thrall et al. 2012). Moreover, field studies have shown that common clones of a gynogenetic fish were more infected by digenetic trematodes when compared to coexisting sexual fish, unless the sexual lineages were highly inbred (Lively et al. 1990). Similarly, the most common asexual clones (as determined by genotype at MHC loci) were more infected than the sexual individuals in the fish Carassius gibelio (Šimková et al. 2013). In addition, common clones of the host plant Chondrilla juncea, were found to be more infected than rare clones by a rust fungus (Puccinia chondrilla) across a broad geographic range in eastern Turkey (Chaboudez and Burdon 1995). Finally, asexual flatworms (Schmidtea polychroa) were more infected by an amoeboid protozoan than sexual individuals (Michiels et al. 2001). Hence, the basic idea (frequency-dependent selection) at the core of the Red Queen hypothesis has been supported across a wide variety of study systems.

In summary, our results show that asexual females were more infected than sexual females in 4 years of a 5 -year study on a freshwater snail in a natural glacial lake population. Given that infected individuals are sterilized, the greater infection of asexuals would help to overcome the cost of producing males in the sexual population and accordingly may explain the stable coexistence of sexual and asexual forms of reproduction. In addition, our results suggest that long-term field studies are required to fully document the complicated, spatiotemporal evolutionary dance of host-parasite coevolution and the evolutionary stability of sex.

\section{Acknowledgments}

We thank J. Van Berkel for his assistance at the University of Canterbury field station, and we thank L. Delph, M. Gibson, and an anonymous reviewer for extensive comments on the manuscript. The authors acknowledge financial support from National Science Foundation grants DEB-0640639 to C.M.L. and J. J. and DDIG-1110437 to D.V., the Swiss National Science Foundation to J.J., and a 
National Geographic Society (883510) award to D.V. We also thank the American Society of Naturalists for supporting the symposium entitled "The Ecology, Evolution and Coevolution of Host-Parasite Interactions," which was held in Snowbird, Utah, in 2013.

\section{Literature Cited}

Agrawal, A. F., and C. M. Lively. 2001. Parasites and the evolution of self fertilization. Evolution 55:869-879.

Antonovics, J., and N. C. Ellstrand. 1984. Experimental studies of the evolutionary significance of sexual reproduction. I. A test of the frequency-dependent selection hypothesis. Evolution 38:103-115.

Bell, G. 1982. The masterpiece of nature: the evolution and genetics of sexuality. University of California Press, Berkeley.

Buckling, A., and P. B. Rainey. 2002. Antagonistic coevolution between a bacterium and a bacteriophage. Proceedings of the Royal Society B: Biological Sciences 269:931-936.

Chaboudez, P., and J. J. Burdon. 1995. Frequency-dependent selection in a wild plant-pathogen system. Oecologia (Berlin) 102:490-493.

Decaestecker, E., S. Gaba, J. A. M. Raeymaekers, R. Stoks, L. Van Kerckhoven, D. Ebert, and L. De Meester. 2007. Host-parasite "Red Queen" dynamics archived in pond sediment. Nature 450:870-874.

Doncaster, C. P., G. E. Pound, and S. J. Cox. 2000. The ecological cost of sex. Nature 404:281-285.

Duncan, A., and T. J. Little. 2007. Parasite-driven genetic change in a natural population of Daphnia. Evolution 64:796-803.

Duneau, D., P. Luijckx, F. Ben-Ami, C. Laforsch, and D. Ebert. 2011. Resolving the infection process reveals striking differences in the contribution of environment, genetics and phylogeny to host-parasite interactions. BMC Biology 9:11-22.

Dybdahl, M., J. Jokela, L. F. Delph, B. Koskella, and C. M. Lively. 2008. Hybrid fitness in a locally adapted parasite. American Naturalist 172:772-782.

Dybdahl, M. F., and C. M. Lively. 1995. Diverse, endemic and polyphyletic clones in mixed populations of a freshwater snail (Potamopyrgus antipodarum). Journal of Evolutionary Biology 8:385-398.

1998. Host-parasite coevolution: evidence for rare advantage and time-lagged selection in a natural population. Evolution 52: 1057-1066.

Fischer, O., and P. Schmid-Hempel. 2005. Selection by parasites may increase host recombination frequency. Biology Letters 1:193-195.

Fox, J. A., M. F. Dybdahl, J. Jokela, and C. M. Lively. 1996. Genetic structure of coexisting sexual and clonal subpopulations in a freshwater snail (Potamopyrgus antipodarum). Evolution 50:1541-1548.

Gandon, S., and S. P. Otto. 2007. The evolution of sex and recombination in response to abiotic or coevolutionary fluctuations in epistasis. Genetics 175:1835-1853.

Haldane, J. B. S. 1949. Disease and evolution. La Ricerca Scientifica 19(suppl.):68-76.

Hamilton, W. D. 1982. Pathogens as causes of genetic diversity in their host populations. Pages 269-296 in R. M. Anderson and R. M. May, eds. Population biology of infectious diseases. New York, Springer.

Hechinger, R. F. 2012. Faunal survey and identification key for the trematodes (Platyhelminthes: Digenea) infecting Potamopyrgus antipodarum (Gastropoda: Hydrobiidae) as first intermediate host. Zootaxa 3418:1-27.
Howard, R. S., and C. M. Lively. 1994. Parasitism, mutation accumulation and the maintenance of sex. Nature 367:554-557.

Jaenike, J. 1978. A hypothesis to account for the maintenance of sex within populations. Evolutionary Theory 3:191-194.

Jokela, J., M. F. Dybdahl, and C. M. Lively. 1999. Habitat-specific variation in life-history traits, clonal population structure, and parasitism in a freshwater snail (Potamopyrgus antipodarum). Journal of Evolutionary Biology 12:350-360.

Jokela, J., M. F. Dybdahl, and C. M. Lively. 2009. The maintenance of sex, clonal dynamics, and host-parasite coevolution in a mixed population of sexual and asexual snails. American Naturalist 174(suppl.):S43-S53.

Jokela, J., and C. M. Lively. 1995a. Parasites, sex, and early reproduction in a mixed population of freshwater snails. Evolution 49: $1268-1271$

- $1995 \mathrm{~b}$. Spatial variation for infection by digenetic trematodes in a population of freshwater snails (Potamopyrgus antipodarum). Oecologia (Berlin) 103:509-517.

Jokela, J., C. M. Lively, M. F. Dybdahl, and J. A. Fox. 1997. Evidence for a cost of sex in the freshwater snail Potamopyrgus antipodarum. Ecology 78:452-460.

- 2003. Genetic variation in sexual and clonal lineages of a freshwater snail. Biological Journal of the Linnean Society 79:165181.

Kelley, S. E., J. Antonovics, and J. Schmitt. 1988. A test of the shortterm advantage of sexual reproduction. Nature 331:714-716.

Kerstes, N. A. G., C. Berenos, P. Schmid-Hempel, and K. M. Wegner. 2012. Antagonistic experimental coevolution with a parasite increases host recombination frequency. BMC Evolutionary Biology 12:18.

King, K. C., L. F. Delph, J. Jokela, and C. M. Lively. 2009. The geographic mosaic of sex and the Red Queen. Current Biology 19: 1438-1441.

- 2011. Coevolutionary hotspots and coldspots for host sex and parasite local adaptation in a snail-trematode interaction. Oikos 120:1335-1340.

Koskella, B. 2013. Phage-mediated selection on microbiota of a longlived host. Current Biology 23:1256-1260.

2014. Bacteria-phage interactions across time and space: merging local adaptation and time-shift experiments to understand parasite evolution. American Naturalist 184(suppl.):S9-S21.

Koskella, B., and C. M. Lively. 2009. Evidence for negative frequencydependent selection during experimental coevolution of a freshwater snail and a sterilizing tremotode. Evolution 63:2213-2221.

Koskella, B., D. Vergara, and C. M. Lively. 2011. Experimental evolution of sexual host populations in response to sterilizing parasites. Evolutionary Ecology Research 13:315-322.

Lively, C. M. 1987. Evidence from a New Zealand snail for the maintenance of sex by parasitism. Nature 328:519-521.

- 1992. Parthenogenesis in a freshwater snail: reproductive assurance versus parasitic release. Evolution 46:907-913.

- 2009. The maintenance of sex: host-parasite coevolution with density-dependent virulence. Journal of Evolutionary Biology 22:2086-2093.

- 2010. An epidemiological model of host-parasite coevolution and sex. Journal of Evolutionary Biology 23:1490-1497.

Lively, C. M., C. Craddock, and R. C. Vrijenhoek. 1990. Red Queen hypothesis supported by parasitism in sexual and clonal fish. Nature 344:864-866. 
Lively, C. M., and M. F. Dybdahl. 2000. Parasite adaptation to locally common host genotypes. Nature 405:679-681.

Lively, C. M., and J. Jokela. 2002. Temporal and spatial distributions of parasites and sex in a freshwater snail. Evolutionary Ecology Research 4:219-226.

Lloyd, D. G. 1980. Benefits and handicaps of sexual reproduction. Evolutionary Biology 13:69-111.

Luijckx, P., F. Ben-Ami, L. Mouton, L. Du Pasquier, and D. Ebert. 2011. Cloning of the unculturable parasite Pasteuria ramosa and its Daphnia host reveals extreme genotype-genotype interactions. Ecology Letters 14:125-131.

Luijckx, P., H. Fienberg, D. Duneau, and D. Ebert. 2013. A matchingallele model explains host resistance to parasites. Current Biology 23:1085-1088.

Masri, L., R. D. Schulte, N. Timmermeyer, S. Thanisch, L. L. Crummenerl, G. Jansen, N. K. Michiels, and H. Schulenburg. 2013. Sex differences in host defence interfere with parasite-mediated selection for outcrossing during host-parasite coevolution. Ecology Letters 16:461-468.

Maynard Smith, J. 1971. What use is sex? Journal of Theoretical Biology 30:319-335.

- 1978. The evolution of sex. Cambridge University Press, Cambridge.

Michiels, N. K., L. W. Beukeboom, N. Pongratz, and J. Zeitlinger. 2001. Parthenogenetic flatworms have more symbionts than their coexisting, sexual conspecifics, but does this support the Red Queen? Journal of Evolutionary Biology 14:110-119.

Morran, L. T., R. C. Parrish, I. A. Gelarden, and C. M. Lively. 2013. Temporal dynamics of outcrossing and host mortality rates in hostpathogen experimental coevolution. Evolution 67:1860-1869.

Morran, L. T., O. G. Schmidt, I. A. Gelarden, R. C. Parrish, and C. M. Lively. 2011. Running with the Red Queen: host-parasite coevolution selects for biparental sex. Science 333:216-218.

Neiman, M., D. Paczesniak, D. M. Soper, A. T. Baldwin, and G. Hehman. 2011. Wide variation in ploidy level and genome size in a New Zealand freshwater snail with coexisting sexual and asexual lineages Evolution 65:3202-3216.

Norusis, M. J. 1994. SPSS advanced statistics 6.1. SPSS, Chicago.

Orr, H. A. 2009. Fitness and its role in evolutionary genetics. Nature Reviews Genetics 10:531-539.

Osnas, E. E., and C. M. Lively. 2006. Host ploidy, parasitism and immune defence in a coevolutionary snail-trematode system. Journal of Evolutionary Biology 19:42-48.

Paczesniak, D. 2012. Ecological and evolutionary dynamics in natural populations of co-existing sexual and asexual lineages. Dissertation thesis, ETH Zurich.

Paczesniak, D., S. Adolfsson, K. Liljeroos, K. Klappert, C. M. Lively, and J. Jokela. 2014. Faster clonal turnover in high-infection habitats provides evidence for parasite-mediated selection. Journal of Evolutionary Biology 27:417-428.

Peters, A. D., and C. M. Lively. 1999. The Red Queen and fluctuating epistasis: a population genetic analysis of antagonistic coevolution. American Naturalist 154:393-405.

. 2007. Short- and long-term benefits and detriments to recombination under antagonistic coevolution. Journal of Evolutionary Biology 20:1206-1217.
Phillips, N. R., and D. M. Lambert. 1989. Genetics of Potamopyrgus antipodarum (Gastropoda, Prosobranchia): evidence for reproductive modes. New Zealand Journal of Zoology 16:435-445.

Rauch, G., M. Kalbe, and T. B. H. Reusch. 2006. One day is enough: rapid and specific host-parasite interactions in a stickleback-trematode system. Biology Letters 2:382-384.

Schmid-Hempel, P., and J. Jokela. 2002. Socially structured populations and evolution of recombination under antagonistic coevolution. American Naturalist 160:403-408.

Šimková, A., M. Košař, L. Vetešník, and M. Vyskočilová. 2013. MHC genes and parasitism in Carassius gibelio, a diploid-triploid fish species with dual reproduction strategies. BMC Evolutionary Biology 13:122.

Soper, D. M., L. F. Delph, and C. M. Lively. 2012. Multiple paternity in the freshwater snail, Potamopyrgus antipodarum. Ecology and Evolution 2:3179-3185. doi:10.1002/ece3.408.

Thompson, J. N. 1994. The coevolutionary process. University of Chicago Press, Chicago.

. 2005. The geographic mosaic of coevolution. University of Chicago Press, Chicago.

Thrall, P. H., A. L. Laine, M. Ravensdale, A. Nemri, P. N. Dodds, L. G. Barrett, and J. J. Burdon. 2012. Rapid genetic change underpins antagonistic coevolution in a natural host-pathogen metapopulation. Ecology Letters 15:425-435.

Vergara, D., J. Jokela, and C. M. Lively. 2014. Data from: Infection dynamics in coexisting sexual and asexual populations: support for the Red Queen hypothesis. American Naturalist, Dryad Digital Repository, http://dx.doi.org/10.5061/dryad.29nk3.

Vergara, D., C. M. Lively, K. C. King, and J. Jokela. 2013. The geographic mosaic of sex and infection in lake populations of a New Zealand snail at multiple spatial scales. American Naturalist 182: 484-493.

Verhoeven, K. J. F., and A. Biere. 2013. Geographic parthenogenesis and plant-enemy interactions in the common dandelion. BMC Evolutionary Biology 13:23.

Wallace, C. 1992. Parthenogenesis, sex, and chromosomes in Potamopyrgus. Journal of Molluscan Studies 58:93-107.

Wegner, K., C. Berenos, and P. Schmid-Hempel. 2008. Nonadditive genetic components in resistance of the red flour beetle Tribolium castanaeum against parasite infection. Evolution 62:2381-2392.

Wilfert, L., and P. Schmid-Hempel. 2008. The genetic architecture of susceptibility to parasites. BMC Evolutionary Biology 8:187.

Wilson, C. G., and P. W. Sherman. 2010. Anciently asexual bdelloid rotifers escape lethal fungal parasites by drying up and blowing away. Science 327:547-576.

2013. Spatial and temporal escape from fungal parasitism in natural communities of anciently asexual bdelloid rotifers. Proceedings of the Royal Society B: Biological Sciences 280:20131255.

Winterbourn, M. J. 1974. Larval Trematoda parasitizing the New Zealand species of Potamopyrgus (Gastropoda: Hydrobiidae). Mauri Ora 2:17-30.

Wolinska, J., and P. Spaak. 2009. The cost of being common: evidence from natural Daphnia populations. Evolution 63:1893-1901.

Editor: Judith L. Bronstein 